\title{
5D SUSY Orbifold SU(6) GUT and Pseudo-Goldstone Higgs Doublets
}

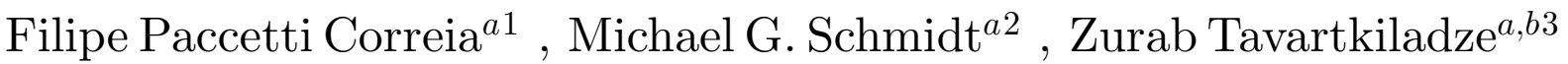 \\ ${ }^{a}$ Institut für Theoretische Physik, Universität Heidelberg, Philosophenweg 16, \\ D-69120 Heidelberg, Germany \\ ${ }^{b}$ Institute of Physics, Georgian Academy of Sciences, Tbilisi 380077, Georgia
}

\begin{abstract}
We construct a 5D SUSY $S U(6)$ GUT on an $S^{(1)} / Z_{2} \times Z_{2}^{\prime}$ orbifold. The first stage of gauge symmetry breaking occurs through compactification and a specific selection of boundary conditions. Additional symmetries play a crucial role for the generation of $\mu$ and $B \mu$ terms of appropriate values: with a $S U(2)_{\text {cus }}$ custodial symmetry the Higgs doublets naturally emerge as massless pseudo-Goldstone bosons in the unbroken SUSY limit. After SUSY breaking they get masses of the order of the weak scale. If instead of $S U(2)_{\text {cus }}$ a discrete $Z_{5}$ symmetry is applied the Higgs doublet's masses are still adequately suppressed, but they are not pseudoGoldstones. The $Z_{5}$ discrete symmetry also can be very important for GUT scale generation and an all order hierarchy. Fermion masses are naturally generated and nicely blend with additional symmetries. In the considered scenario unification of the three gauge couplings occurs near $10^{16} \mathrm{GeV}$.
\end{abstract}

\footnotetext{
${ }^{1}$ E-mail address: F.Paccetti@ThPhys.Uni-Heidelberg.DE

${ }^{2}$ E-mail address: M.G.Schmidt@ThPhys.Uni-Heidelberg.DE

${ }^{3}$ E-mail address: Z.Tavartkiladze@ThPhys.Uni-Heidelberg.DE
} 


\section{Introduction}

The doublet-triplet (DT) splitting problem is an 'Achilles heel' of any GUT. When constructing a realistic model, one has to explain why the doublet components are split in mass from their colored triplet partners, with a ratio $M_{T} / M_{D} \gtrsim 10^{13}$. A big triplet mass is needed for the proton stability and for maintaining successful unification of the three gauge coupling constants. Recently a way was proposed, based on a higher dimensional construction [1], [2]- [4] how to cure various phenomenological problems common to GUTs in an economical way: It was observed that the compactification of a 5D $(N=1)$ SUSY $S U(5)$ model on an $S^{(1)} / Z_{2} \times Z_{2}^{\prime}$ orbifold provides a natural DT splitting, GUT symmetry breaking and proton stability. Furthermore, due to 5D $N=1$ SUSY, the $\mu$-term vanishes at the 5D level. This can be considered as an excellent starting point for realistic model building. However, on the $4 \mathrm{D}$ level, there is no reason to have a vanishing $\mu$-term and some specific extensions [5]-[7] are required to provide a $\mu$-term of appropriate magnitude. One of the attractive ideas for the solution of DT splitting and $\mu$ problems in 4D is the pseudo-Goldstone boson (PGB) mechanism [8]-[11], where Higgs doublets emerge as Goldstone modes of a spontaneously broken global symmetry. If the symmetry of the 'scalar' superpotential is larger than the symmetry of the whole Lagrangian, then due to the symmetry breaking PGBs emerge, which are massless, as stated in the Goldstone theorem. Thanks to SUSY, the superpotential is not renormalized and the PGBs remain massless in the unbroken SUSY limit. After the SUSY breaking their masses will not exceed the SUSY scale $m \sim 1 \mathrm{TeV}$. In this way the DT splitting problem can be resolved. It also turns out that the $\mu$ - and $B \mu$-terms get the right magnitude.

In this paper we consider a SUSY $S U(6)$ GUT in five dimensions. The first stage of GUT symmetry breaking occurs via compactification of one extra dimension, leaving an unbroken $S U(3)_{c} \times S U(3)_{L} \times U(1)$ symmetry on the $4 \mathrm{D}$ level. Then we invoke the custodial $S U(2)_{\text {cus }}$ symmetry [12] in order to get a natural solution of the $\mu$ problem. The breaking of $S U(3)_{L}$ to $S U(2)_{L}$ by the Higgs mechanism requires the existence of Goldstone doublets

in the Higgs sector. Although these are eaten by the massive gauge bosons, there are additional states related to them by the $S U(2)_{\text {cus }}$ symmetry. These will remain massless PGBs in the unbroken SUSY regime and are good candidates for the MSSM doubletantidoublet pair. After SUSY breaking the $B \mu$ and $\mu$ terms are naturally generated and have values of the order of the SUSY scale. We emphasize that some difficulties, present in 4D $S U(6)$ GUTs with custodial $S U(2)_{\text {cus }}$ symmetry mechanism [12 such as the emergence of an undesirable intermediate scale (which disrupts the unification of the three gauge coupling constants) do not exist in our model due to the extra-dimensional construction. The model is very economical as there is no need for further extensions [13] in order to avoid the difficulties mentioned before and to make the $S U(2)_{\text {cus }}$ symmetry mechanism [12] efficient. We also present a discussion about the emergence of the $S U(2)_{\text {cus }}$ symmetry 
in the theory. An alternative model is constructed, in which $S U(2)_{\text {cus }}$ is replaced by a $\mathcal{Z}_{5}$ discrete symmetry. The latter provides an all order hierarchy and natural generation of the GUT scale $M_{G} \sim 10^{16} \mathrm{GeV}$. The generation of fermion masses and the unification of the gauge coupling constants are also studied.

\section{5D SUSY $S U(6)$ on $S^{(1)} / Z_{2} \times Z_{2}^{\prime}$ orbifold}

Consider a $5 \mathrm{D} N=1$ SUSY $S U(6)$ GUT where the 5 th dimension is a $S^{(1)} / Z_{2} \times Z_{2}^{\prime}$ orbifold. By a suitable assignment of the orbifold $Z_{2} \times Z_{2}^{\prime}$ parities, $\left(P, P^{\prime}\right)=( \pm, \pm)$, to the different components of the gauge fields, it is possible to break the gauge symmetry and half of the supersymmetries at a fixed point. The 5D $N=1$ gauge supermultiplet is, from a $4 D$ point of view, a $N=2$ supermultiplet $V_{N=2}$, which contains a $4 \mathrm{D} N=1$ gauge superfield $V$ and a chiral superfield $\Sigma$, both in the adjoint representation 35 of $S U(6): V_{N=2}=(V, \Sigma)$. We select boundary conditions in such a way as to break $S U(6)$ to $S U(3)_{c} \times S U(3)_{L} \times U(1) \equiv G_{331}$. It will turn out that this breaking (through the $G_{331}$ channel) is crucial if one wants to obtain the MSSM Higgses as PGB modes after a second stage of symmetry breaking. In terms of $G_{331}$ the decomposition of $V(35)$ reads

$$
V(35)=V_{c}(8,1)_{0}+V_{3 L}(1,8)_{0}+V_{U(1)}(1,1)_{0}+V_{c \bar{L}}(3, \overline{3})_{2}+V_{\bar{c} L}(\overline{3}, 3)_{-2},
$$

and similar for $\Sigma(35)$. The subscripts in eq.(1) denote $U(1)$ hypercharges in $1 / \sqrt{12}$ units:

$$
Y_{U(1)}=\frac{1}{\sqrt{12}}(1,1,1,-1,-1,-1)
$$

We choose the $Z_{2} \times Z_{2}^{\prime}$ parities of the fragments of $V$ and $\Sigma$ as

$$
\begin{gathered}
\left(V_{c}, V_{3 L}, V_{U(1)}\right) \sim(+,+), \quad\left(V_{c \bar{L}}, V_{\bar{c} L}\right) \sim(-,+), \\
\left(\Sigma_{c}, \Sigma_{3 L}, \Sigma_{U(1)}\right) \sim(-,-), \quad\left(\Sigma_{c \bar{L}}, \Sigma_{\bar{c} L}\right) \sim(+,-),
\end{gathered}
$$

so that at the $y=0$ fixed point we have $4 \mathrm{D} N=1$ SUSY and $G_{331}$ gauge symmetry.

\section{$2.1 \quad G_{331}$ breaking and pseudo-Goldstone Higgses}

For the further breaking of $G_{331}$ down to the standard model group $S U(3)_{c} \times S U(2)_{L} \times$ $U(1)_{Y} \equiv G_{321}$, we introduce the following Higgses on the 5D level

$$
\mathcal{H}_{N=2}^{(i)}(6)=(\mathcal{H}, \overline{\mathcal{H}})^{(i)}, \quad \mathcal{H}_{N=2}^{\prime}{ }^{(i)}(6)=\left(\mathcal{H}^{\prime}, \overline{\mathcal{H}}^{\prime}\right)^{(i)}, \quad i=1,2,
$$


where $\mathcal{H}^{(i)}, \mathcal{H}^{\prime(i)}$ and $\overline{\mathcal{H}}^{(i)}, \overline{\mathcal{H}}^{\prime(i)}$ are in the representations $\mathbf{6}$ and $\overline{\mathbf{6}}$ of $S U(6)$, resp. In terms of $G_{331}, \mathcal{H}^{(i)}$ and $\overline{\mathcal{H}}^{(i)}$ decompose as

$$
\mathcal{H}^{(i)}=T^{(i)}(3,1)_{1}+H^{(i)}(1,3)_{-1}, \quad \overline{\mathcal{H}}^{(i)}=\bar{T}^{(i)}(\overline{3}, 1)_{-1}+\bar{H}^{(i)}(1, \overline{3})_{1},
$$

and similar for $\mathcal{H}^{\prime(i)}$ and $\overline{\mathcal{H}}^{\prime(i)}$. With the $Z_{2} \times Z_{2}^{\prime}$ parities

$$
\begin{gathered}
\left(H^{(i)}, \bar{H}^{\prime(i)}\right) \sim(+,+), \quad\left(\bar{H}^{(i)}, H^{\prime(i)}\right) \sim(-,-), \\
\left(T^{(i)}, \bar{T}^{\prime(i)}\right) \sim(-,+), \quad\left(\bar{T}^{(i)}, T^{\prime(i)}\right) \sim(+,-)
\end{gathered}
$$

we have two $S U(3)_{L}$ triplet-antitriplet pairs $H^{(i)}, \bar{H}^{\prime(i)}$ at the $y=0$ fixed point . The $S U(3)_{c}$ triplets $T^{(i)}, T^{\prime(i)}, \bar{T}^{(i)}, T^{\prime(i)}$ have no zero modes. The $H$ fields can be used to break $S U(3)_{L} \times U(1)$ down to $S U(2)_{L} \times U(1)_{Y}$. Note that for this purpose it is sufficient to have one $S U(3)_{L}$ triplet-antitriplet pair, which we choose to be $H^{(1)}, \bar{H}^{\prime(1)}$. If the third components of these triplets get non zero 'scalar' VEVs, then the breaking $S U(3)_{L} \times U(1) \rightarrow S U(2)_{L} \times U(1)_{Y}$ occurs. Consequently, the $S U(2)_{L}$ doublet and antidoublet components of $H^{(1)}$ and $\bar{H}^{\prime(1)}$ resp. are absorbed by the appropriate gauge fields and therefore are genuine Goldstones. But we have an additional pair of states, $H^{(2)}$, $\bar{H}^{\prime(2)}$, with precisely the same transformation properties as $H^{(1)}, \bar{H}^{\prime(1)}$. If the $H^{(2)}$ and $\bar{H}^{\prime(2)}$ states are related to the $H^{(1)}$ and $\bar{H}^{\prime(1)}$ resp. by some specific symmetry, then the unabsorbed physical doublets might be also massless. If some care is taken in this procedure and they are massless at the tree level, then due to SUSY they will be protected from getting a radiative mass and turn out to be massless pseudo-Goldstones in the unbroken SUSY limit. To realize this, we introduce a custodial symmetry, $S U(2)_{\text {cus }}$, under which $H^{(1)}, H^{(2)}$ form a doublet $\left(H^{(1)}, H^{(2)}\right) \equiv H_{m}$ [where $m=1,2$ is $S U(2)_{\text {cus }}$ index] and $\bar{H}^{\prime(1)}, \bar{H}^{\prime(2)}$ - an antidoublet $\left(\bar{H}^{\prime(1)}, \bar{H}^{\prime(2)}\right) \equiv \bar{H}^{m}$. The component structure of $\bar{H}^{m}, H_{m}$ can schematically be represented as

$$
H_{m}=\left(\begin{array}{cc}
D_{1} & D_{2} \\
\chi_{1} & \chi_{2}
\end{array}\right), \quad \bar{H}^{m}=\left(\begin{array}{cc}
\bar{D}^{1} & \bar{D}^{2} \\
\bar{\chi}^{1} & \bar{\chi}^{2}
\end{array}\right) .
$$

where $D, \bar{D}$ are doublet-antidoublets of $S U(2)_{L}$ and $\chi, \bar{\chi}$ (third components of $H, \bar{H}$ ) are $S U(2)_{L}$ singlets. With VEVs

$$
\left\langle H_{m}\right\rangle=\left(\begin{array}{cc}
0 & 0 \\
v_{0} & 0
\end{array}\right), \quad\left\langle\bar{H}^{m}\right\rangle=\left(\begin{array}{cc}
0 & 0 \\
v_{0} & 0
\end{array}\right),
$$


the $S U(3)_{L} \times U(1)$ will be broken down to $S U(2)_{L} \times U(1)_{Y}$, where

$$
Y=\frac{2}{\sqrt{5}} Y_{U(1)}-\frac{1}{\sqrt{5}} Y_{U(1)^{\prime}} .
$$

$Y_{U(1)}$ is given in (2) and $Y_{U(1)^{\prime}}$ corresponds to the $S U(3)_{L}$ 's broken generator

$$
Y_{U(1)^{\prime}}=\frac{1}{\sqrt{12}} \operatorname{Diag}(1,1,-2) .
$$

Let us now demonstrate more precisely how the mechanism works. For this purpose we will write $4 \mathrm{D}$ brane couplings. We also introduce a singlet superfield $S$ with zero mode state 1 . As the relevant renormalizable 4D 'scalar' superpotential, invariant under the $G_{331} \times S U(2)_{\text {cus }}$ symmetry, we take

$$
W=\bar{H}^{m}\left(M_{H}+\lambda S\right) H_{m}+\frac{1}{2} M_{S} S^{2}+\frac{1}{3} \sigma S^{3},
$$

where $M_{H}, M_{S}$ are mass scales and $\lambda, \sigma$ are dimensionless couplings. With (8) all $D$-terms automatically vanish, while the conditions $F_{\bar{H}^{m}}=F_{H_{m}}=F_{S}=0$ give us

$$
\langle S\rangle \equiv S_{0}=-\frac{M_{H}}{\lambda}, \quad v_{0}^{2}=\frac{M_{H}}{\lambda}\left(M_{S}-\frac{\sigma}{\lambda} M_{H}\right)
$$

With (12) and (7), (8) it is easy to see from (11) that the superpotential masses of $D_{m}, \bar{D}^{m}$ doublet superfields vanish: $M_{D}=M_{H}+\lambda\langle S\rangle=0$. Let's consider the mass spectrum for the scalar and the fermionic doublet components separately. As we have already seen, none of them gets mass from the superpotential. On the other hand we must take into account the $S U(3)_{L} D$-terms contribution to the Higgs doublets potential. The state $\frac{1}{\sqrt{2}}\left(D_{1}+\bar{D}^{1 *}\right)_{s}$ [the subscript 's' refers to the scalar component of appropriate superfield] is the massless genuine Goldstone boson absorbed by the doublet-antidoublet gauge bosons, while the states $\frac{1}{\sqrt{2}}\left(\bar{D}^{1 *}-D_{1}\right)_{s}$ acquire the mass $g_{S U(3)_{L}} v_{0}$ from the $D$-terms $\left(g_{S U(3)_{L}}\right.$ denotes the $S U(3)_{L}$ gauge coupling constant). Of course the same happens with their fermionic partners: they get the mass $g_{S U(3)_{L}} v_{0}$ through the mixing with the $S U(2)_{L}$ doublet-antidoublet gauginos. This is indeed the supersymmetric Higgs mechanism (i.e. the discussion can be applied to complete superfields, also including their auxiliary fields). As far as the second pair of doublets, $D_{2}, \bar{D}^{2}$, is concerned, they are related to $D_{1}, \bar{D}^{1}$ by the $S U(2)_{\text {cus }}$ symmetry and thus their masses from the superpotential (11) are zero. Therefore, we will identify them with the MSSM pair of doublets

$$
D_{2} \equiv h_{u}, \quad \bar{D}^{2} \equiv h_{d}
$$

\footnotetext{
${ }^{4}$ On the $5 \mathrm{D}$ level one can have a singlet state $\mathcal{S}_{N=2}=(S, \bar{S})$ and the following orbifold parities for its fragments: $S \sim(+,+), \bar{S} \sim(-,-)$.
} 
Note that $h_{u}$ and $h_{d}$ are massless PGBs. For this, the $S U(2)_{\text {cus }}$ symmetry is crucial, which forbids the mixing terms $M_{12} \bar{H}^{1} H_{2}, M_{21} \bar{H}^{2} H_{1}$ in the superpotential. These couplings would destroy the hierarchy since $M_{12} \sim M_{21} \gg \mathcal{O}(100 \mathrm{GeV})$.

Before we turn our attention to the issue of SUSY breaking, let us comment on the singlet states coming from $H_{m}, \bar{H}^{m}$ [see (7)] and $S$. The scalar component $\operatorname{Im}\left(\bar{\chi}^{1}-\chi_{1}\right)_{s}$ is a genuine Goldstone eaten up by the gauge field which corresponds to the broken Abelian factor, while the superposition $\operatorname{Re}\left(\chi_{1}-\bar{\chi}^{1}\right)_{s}$ gets mass $2 g_{S U(3)_{L}}^{2} v_{0}^{2}$ from the $D$ terms. Other scalar singlets from $\bar{\chi}^{1}, \chi_{1}$ and $S$ get masses of the order of $M_{G}$. The fermionic superpartner's mass spectrum looks similarly due to SUSY. As far as the states $\chi_{2}, \bar{\chi}^{2}$ are concerned, they remain massless in unbroken SUSY limit. This spectrum of particles can be also explained with symmetry arguments. Looking on (11) one can verify that the $S U(2)_{\text {cus }}$ symmetry, which acts on $\bar{H}^{m}, H_{m}$, gives rise to an accidental $U(6)_{G l}$ symmetry in the superpotential. The VEVs in (8) break $U(6)_{G l}$ down to $U(5)_{G l}$ and the number of Goldstones is $N_{G o l}=36-25=11$. At the same time, the same VEVs break the $S U(3)_{L} \times U(1)$ local symmetry down to $S U(2)_{L} \times U(1)_{Y}$ and therefore the number of genuine Goldstones is $N_{G G o l}=8+1-(3+1)=5$. So, from eleven $\left(=N_{G o l}\right)$ Goldstones only five $\left(=N_{G G o l}\right)$ are absorbed and the remaining six are pseudo-Goldstones. Amongst them four correspond to the doublet-antidoublet pair $h_{u}, h_{d}$ and the remaining two are the MSSM singlets $\chi_{2}, \bar{\chi}^{2}$. Of course, the $D$-terms do not have $U(6)_{G l}$ symmetry, but they do not contribute to the pseudo-Goldstone masses unless SUSY is broken.

\section{SUSY breaking: generation of $B \mu$ and $\mu$}

Throughout our discussions above we assumed that SUSY is unbroken. We now investigate the effects of SUSY breaking. As we will see, the inclusion of soft SUSY breaking terms automatically generates $B \mu$ and $\mu$ terms of the order of the SUSY breaking scale $\left(\sim m^{2}\right.$ and $m$ resp.). Let us thus take all soft SUSY breaking terms (for $H_{m}, \bar{H}^{m}$ and $S$ ) in the potential

$$
\begin{gathered}
V_{\text {soft }}=m \frac{2}{H}\left|\bar{H}^{m}\right|^{2}+m_{H}^{2}\left|H_{m}\right|^{2}+m_{s}^{2}|S|^{2}+A_{1} m\left[\bar{H}^{m}\left(M_{H}^{\prime}+\lambda S\right) H_{m}+\text { h.c. }\right]+ \\
A_{2} m\left(\frac{1}{2} M_{S}^{\prime} S^{2}+\frac{1}{3} \sigma S^{3}+\text { h.c. }\right)
\end{gathered}
$$

[In (14) all fields denote the scalar components of the associated superfields]. (14) is the most general soft SUSY breaking potential, where neither universality nor proportionality are assumed. The masses $m_{\bar{H}}, m_{H}, m_{s}$ are of the order of the SUSY scale $m \sim 1 \mathrm{TeV}$, while $M_{H}^{\prime} \sim M_{H}$ and $M_{S}^{\prime} \sim M_{S}$. We also assume that the dimensionless couplings $A_{1}$, $A_{2}$ are of the order of one. The complete potential has the form

$$
V=\left|F_{\bar{H}^{m}}\right|^{2}+\left|F_{H_{m}}\right|^{2}+\left|F_{S}\right|^{2}+V_{\text {soft }}+D \text { - terms },
$$

where the $F$-terms must be derived from superpotential (11). The inclusion of soft terms will only slightly shift the solutions (12), because we assume that $M_{H}, M_{S} \gg m$. We will 
search, therefore, for VEV solutions of extrema of (15), in the form

$$
\langle S\rangle=S_{0}(1+x), \quad\left\langle\chi_{1}\right\rangle \equiv v=v_{0}(1+y), \quad\left\langle\bar{\chi}^{1}\right\rangle \equiv \bar{v}=v_{0}(1+z)
$$

where $S_{0}, v_{0}$ are given in (12) and $x, y, z \ll 1$. Different soft masses $m_{H}^{2}, m_{\bar{H}}^{2}$ cause different values for $v, \bar{v}$. Due to this, $D$-terms will not vanish anymore. In fact, $x, y, z$ must be expressed as powers of $m / M$, where $M$ is a mass scale close to $M_{H}, M_{S}$. After a straightforward analysis we find

$$
\begin{gathered}
x=\frac{m}{2 M_{H}}\left(A_{1}-\frac{A_{1}}{\lambda v_{0}^{2}}\left(M_{S}+2 \sigma S_{0}\right)\left(M_{H}^{\prime}+\lambda S_{0}\right)+\frac{A_{2}}{\lambda v_{0}^{2}}\left(M_{S}^{\prime} S_{0}+\sigma S_{0}^{2}\right)\right)+\cdots, \\
y=-\frac{S_{0}}{2 \lambda v_{0}^{2}}\left(M_{S}+2 \sigma S_{0}\right) x-\frac{A_{1}}{2 \lambda^{2} v_{0}^{2}}\left(M_{H}^{\prime}+\lambda S_{0}\right) m+\frac{m_{\bar{H}}^{2}-m_{H}^{2}}{8 \bar{g}^{2} v_{0}^{2}}+\cdots, \\
z=-\frac{S_{0}}{2 \lambda v_{0}^{2}}\left(M_{S}+2 \sigma S_{0}\right) x-\frac{A_{1}}{2 \lambda^{2} v_{0}^{2}}\left(M_{H}^{\prime}+\lambda S_{0}\right) m-\frac{m_{H}^{2}-m_{H}^{2}}{8 \bar{g}^{2} v_{0}^{2}}+\cdots,
\end{gathered}
$$

where $\bar{g}^{2}=\frac{g_{3 L}^{2}}{3}+\frac{g_{1}^{2}}{4}$ and dots in (17) stand for higher powers of $m / M$ which are irrelevant for our studies. Since after SUSY breaking the VEV of $S$ is shifted by the amount $S_{0} x$, in the superpotential (11) there is no precise cancellation of the physical Higgs doublet superfield [defined by (13)] masses anymore. This causes the generation of a $\mu$ term

$$
\mu=\lambda S_{0} x=-M_{H} x \sim m
$$

For the same reason a $B \mu$ term is generated. From (15), taking into account (12), (16), (17) we obtain

$$
B \mu=-\frac{1}{2}\left(m_{H}^{2}+m_{\bar{H}}^{2}\right)-M_{H}^{2} x^{2} \sim m^{2} .
$$

Also, the states $h_{u s}, h_{d s}$ get 'direct' masses through (15). The mass matrix for scalar components of $h_{u}, h_{d}$ is

$$
\begin{gathered}
h_{u_{s}^{*}}^{*} \\
h_{u_{s}} \\
h_{d_{s}^{*}}^{*}
\end{gathered}\left(\begin{array}{cc}
m_{1}^{2} & m_{3}^{2} \\
m_{3}^{2} & m_{2}^{2}
\end{array}\right),
$$

where

$$
\begin{gathered}
m_{1}^{2}=m_{H}^{2}+M_{H}^{2} x^{2}-2 \bar{g}^{2} v_{0}^{2}(z-y), \\
m_{2}^{2}=m_{\bar{H}}^{2}+M_{H}^{2} x^{2}+2 \bar{g}^{2} v_{0}^{2}(z-y), \quad m_{3}^{2}=B \mu .
\end{gathered}
$$

are of the order of $m^{2}$. 
If SUSY breaking occurs through minimal $N=1$ SUGRA [14 at tree level we have $m_{H}^{2}=m_{\bar{H}}^{2}$ and according to (17) $y=z$. This means that $D$-terms do not contribute in Higgs masses and from (19), (21) we have $m_{1}^{2}=m_{2}^{2}=-m_{3}^{2}$ which means that the determinant of matrix (20) is zero. The state $\frac{1}{\sqrt{2}}\left(h_{d}^{*}-h_{u}\right)_{s}$ has mass $2 m_{1}^{2} \sim m^{2}$, while the superposition $\frac{1}{\sqrt{2}}\left(h_{u}+h_{d}^{*}\right)_{s}$ is massless at the tree level. The latter will get a mass through radiative corrections [15], with the dominant contributions coming from top-stop loops. Radiative electro-weak symmetry breaking (EWSB) within PGB scenarios where studied in [11]. In our scenario due to a peculiarity of the model, a shift between soft masses $m_{H}^{2}$ and $m_{\bar{H}}^{2}$ is already sufficient to have an appropriate EWSB. The reason for this is that the difference of $m_{H}^{2}$ and $m_{\bar{H}}^{2}$ causes $y \neq z$ and $D$-terms contribute to the Higgs masses. However, taking into account the solutions (17) of extremum equations, it is easy to see that the contribution from $D$ terms to the determinant is negative. More precisely, taking into account (17), (19), (21) we can see that $m_{1}^{2} m_{2}^{2}-m_{3}^{4}=-\frac{g_{3 L}^{4}}{16 \bar{g}^{4}}\left(m_{H}^{2}-m_{\bar{H}}^{2}\right)^{2}<0$ and the minimum condition is satisfied. For the latter, as we have already mentioned, $m_{H}^{2} \neq m_{\bar{H}}^{2}$ is crucial. This can be achieved by renormalization between the high scale (at which soft terms are universal) and the SUSY scale. Therefore EWSB can naturally occur within our PGB scenario.

\subsection{Symmetries, higher order operators and all order hierar- chy}

For the scenario discussed above the $S U(2)_{\text {cus }}$ symmetry was crucial. One may think about the origin of this symmetry. It can be obtained either accidentally or might have a gauge origin. For example, with two discrete symmetries $\mathcal{Z}_{2}^{(1,2)}$ which are acting as $\mathcal{Z}_{2}^{(1)}:\left(\bar{H}^{1}, H_{1}\right) \rightarrow-\left(\bar{H}^{1}, H_{1}\right), \mathcal{Z}_{2}^{(2)}:\left(\bar{H}^{2}, H_{2}\right) \rightarrow-\left(\bar{H}^{2}, H_{2}\right)$, by imposing the exchange symmetry $\mathcal{S}_{2}$ : $\mathcal{Z}_{2}^{(1)} \rightleftarrows \mathcal{Z}_{2}^{(2)}, \bar{H}^{1} \rightleftarrows \bar{H}^{2}, H_{1} \rightleftarrows H_{2}$, the renormalizable superpotential will have precisely the form (11).

$S U(2)_{\text {cus }}$ can be also gauged. In fact $S U(6) \times S U(2)$ is one of the maximal subgroups of $E_{6}$ and one can assume that at high scales the $E_{6}$ is broken to the $5 D S U(6) \times S U(2)_{\text {cus }}$ gauge group (either by compactification of an additional extra dimension or by some other mechanism) and then $S U(6)$ reduces to $G_{331}$. As we see, the origin of $S U(2)_{\text {cus }}$ symmetry can be different. Important is the form of the superpotential (11), which guarantees naturally light Higgs doublets.

Let us also discuss the higher order operators and their effects. It is easy to verify that non-renormalizable terms of $\left(\bar{H}^{m} H_{n}\right)\left(\bar{H}^{n} H_{m}\right)$ would be dangerous for the hierarchy since the cancellation of doublet's masses would not occur anymore. The reason for this is that these terms explicitly violate the $U(6)_{G l}$ global symmetry (the role of this symmetry was discussed above), which arises accidentally at the renormalizable level. To avoid 
quartic non-renormalizable terms, some discrete symmetries can be applied. These can even provide an all order hierarchy. If instead of $S U(2)_{\text {cus }}$ one introduces a $\mathcal{Z}_{5}$ symmetry acting as $H_{1,2} \rightarrow H_{1,2}, \bar{H}^{1,2} \rightarrow \omega \bar{H}^{1,2}\left(\omega=e^{\mathrm{i} \frac{2 \pi}{5}}\right)$, then the lowest allowed superpotential couplings will be

$$
W^{\prime}=M_{P l}^{3}\left(\frac{\bar{H}^{m} H_{n}}{M_{P l}^{2}}\right)^{5},
$$

where all possible contractions of $S U(6)$ indeces are assumed and $M_{P l}\left(\simeq 2.4 \cdot 10^{18} \mathrm{GeV}\right)$ is the reduced Planck mass. In the unbroken SUSY limit $(22)$ gives $\left\langle\bar{H}^{m}\right\rangle=\left\langle H_{n}\right\rangle=0$. However, it is easy to verify that the inclusion of soft SUSY breaking terms $m \frac{2}{H^{m}}\left|\bar{H}^{m}\right|^{2}+$ $m_{H_{m}}^{2}\left|H_{m}\right|^{2}+A^{\prime} m W^{\prime}$ will give the non-zero solutions $v \sim \bar{v} \sim M_{P l}\left(\frac{m}{M_{P l}}\right)^{1 / 8}$ which for $m \sim 1 \mathrm{TeV}$ are indeed $\sim 10^{16} \mathrm{GeV}$. With these VEVs and the operators in (22) the

Higgs doublet pair has masses $\sim M_{P l}\left(\frac{\bar{v} \cdot v}{M_{P l}^{2}}\right)^{4} \sim m$ of the wanted size. Note that in this case, since the couplings in (22) are strongly suppressed, there is no need for a $S U(2)_{\text {cus }}$ symmetry. $\mathcal{Z}_{5}$ guarantees the generation of non-zero $v, \bar{v}$ VEVs and an all order hierarchy. However, in this case the light Higgs doublets are not the pseudo-Goldstones. This distinguishes this case from the model with an $S U(2)_{\text {cus }}$ symmetry.

As we have seen, the orbifold constructed $S U(6)$ GUT looks very promising for obtaining a nice hierarchy and for solving the $\mu$ problem. In the next section we will show that within the orbifold $S U(6)$ GUT, extended either with $S U(2)_{\text {cus }}$ or by $\mathcal{Z}_{5}$ symmetry, the fermion masses are naturally generated.

\section{Quark-lepton masses}

In order to construct the fermion sector we will introduce some $S U(6)$ states at the 5D level. We will consider here just the case of one generation. The generalization to three families of quark-leptons will be straightforward.

Introduce the following states

$$
\begin{gathered}
\Psi_{N=2}(15)=(15, \overline{15}), \quad \Psi_{N=2}^{\prime}(15)=\left(15^{\prime}, \overline{15}^{\prime}\right) \\
F_{N=2}^{(i)}(6)=(\overline{6}, 6)^{(i)}, \quad F_{N=2}^{\prime}{ }^{(i)}(6)=\left(\overline{6}^{\prime}, 6^{\prime}\right)^{(i)}, \quad i=1,2,
\end{gathered}
$$

where states with primes are so-called copies and are necessary if we want to introduce matter fields in the bulk (see $1^{\text {st }}$ and $3^{\text {rd }}$ ref. in [3] ). Two sets of sextets $(i=1,2)$ are necessary to construct an anomaly free $S U(6)$ orbifold model. In terms of $G_{331}, 15$ and $\overline{6}$ decompose as

$$
15=u^{c}(\overline{3}, 1)_{2}+Q(3,3)_{0}+E^{c}(1, \overline{3})_{-2},
$$




$$
\overline{6}=d^{c}(\overline{3}, 1)_{-1}+\mathcal{L}(1, \overline{3})_{1} .
$$

The decomposition of $15^{\prime}$ and $\overline{6}^{\prime}$ is similar. Since states $\overline{15}$ and 6 are conjugates of 15 and $\overline{6}$ resp. their decompositions also will be conjugate with respect to (24). In terms of $G_{321}$ the states $Q, E^{c}, \mathcal{L}$ read

$$
Q=\left(q, \bar{D}^{c}\right), \quad E^{c}=\left(e^{c}, \bar{L}\right), \quad \mathcal{L}=(l, \xi),
$$

where $q, e^{c}$ and $l$ carry precisely the same quantum numbers as the left handed quark doublet, right handed lepton and left handed lepton doublet, resp. $\bar{D}^{c}$ and $\bar{L}$ have quantum numbers conjugate to the right handed down quark and left handed doublet, resp. $\xi$ is a MSSM singlet. We ascribe the following $Z_{2} \times Z_{2}^{\prime}$ orbifold parities to the states

$$
\left(u^{c}, Q^{\prime}, E^{c}, \mathcal{L}^{(1,2)}, d^{c(1,2)}\right) \sim(+,+), \quad\left(u^{c \prime}, Q, E^{c \prime}, \mathcal{L}^{(1,2)}, d^{c(1,2)}\right) \sim(-,+)
$$

and opposite parities to the mirrors. With the transformations of gauge field fragments given in (3) the 5D SUSY action will be invariant. With the parities in (26), only the states $u^{c}, E^{c}, Q^{\prime}, \mathcal{L}^{(1,2)}, d^{c^{(1,2)}}$ have zero modes. One can easily verify that these states effectively constitute $15+2 \times \overline{6}$ representations of the $S U(6)$ gauge group, which then is free of anomalies. Since at the $y=0$ fixed point we have $4 \mathrm{D} N=1$ SUSY $G_{331}$ symmetry, the brane Yukawa couplings also possess this symmetry. In addition, we also have $S U(2)_{\text {cus }}\left(\right.$ or $\mathcal{Z}_{5}$ ) symmetry. Making the fermion sector consistent with $S U(2)_{\text {cus }}$ we assume that $\mathcal{L}^{(1,2)}$ and $d^{c^{(1,2)}}$ are antidoublets of $S U(2)_{\text {cus }}$

$$
\left(\mathcal{L}^{(1)}, \mathcal{L}^{(2)}\right) \equiv \mathcal{L}^{m}, \quad\left(d^{c /(1)}, d^{c^{\prime \prime}(2)}\right) \equiv d^{c / m}, \quad m=1,2 .
$$

The $G_{331} \times S U(2)_{\text {cus }}$ invariant 4D superpotential Yukawa couplings are

$$
W_{Y}=Q^{\prime} d^{c m} \bar{H}^{n} \epsilon_{m n}+E^{c} \mathcal{L}^{m} \bar{H}^{n} \epsilon_{m n}+\frac{1}{M} Q^{\prime} u^{c} H_{m} H_{n} \epsilon^{m n},
$$

where $\epsilon_{m n}$ is the $S U(2)_{c u s}$ invariant antisymmetric tensor. The first two terms are responsible for the generation of down quark and charged lepton masses, respectively. The last term generates masses for up type quarks. The first two terms in (28) are also crucial for the extra vector-like states to get decoupled. More precisely, substituting appropriate VEVs and extracting appropriate states from $G_{331}$ superfields [see (8), (13), (24), (25)] the first term in (28) gives

$$
Q^{\prime} d^{c \prime m} \bar{H}^{n} \epsilon_{m n} \rightarrow v_{0} \bar{D}^{c} d^{c^{\prime 2}}+q^{\prime} d^{c / 1} \bar{D}^{2}=v_{0} \bar{D}^{c} d^{c^{\prime 2}}+q^{\prime} d^{c 1} h_{d} .
$$

Therefore the state $d^{c^{\prime 2}}$ decouples with $\bar{D}^{c}$ getting a mass $\sim v_{0}$. 目 The states $q^{\prime}, d^{c^{1}}$ have Yukawa coupling (with the down Higgs doublet $h_{d}$ ) and can be identified as left handed

\footnotetext{
${ }^{5}$ For VEVs of $\bar{H}^{1}, H_{1}$ we use $v_{0}$, because the insignificant shift between $v$ and $\bar{v}$ is not relevant for decoupled vector-like states.
} 
quark doublet and right handed down quark resp. The second term in (28) gives

$$
E^{c} \mathcal{L}^{m} \bar{H}^{n} \epsilon_{m n} \rightarrow v_{0} \bar{L} l^{2}+e^{c} l^{1} h_{d}
$$

As we see, $l^{2}$ decouples with $\bar{L}$ forming a massive state. States $e^{c}, l^{1}$ have standard Yukawa couplings (with $h_{d}$ ) and can be identified with the right handed lepton and left handed lepton doublet respectively. Finally, the last term in (28) leads to

$$
\frac{1}{M} Q^{\prime} u^{c} H_{m} H_{n} \epsilon^{m n} \rightarrow \frac{v_{0}}{M} q^{\prime} u^{c} h_{u}
$$

which for $M \sim v_{0} \sim M_{G}$ results in $\lambda_{U} \sim 1$ 6. As we see, the usual Yukawa couplings are obtained in a natural way in a scenario extended with $S U(2)_{\text {cus }}$ symmetry. It is easy to show that phenomenologically required fermion sector can be also built within a model where $S U(2)_{\text {cus }}$ is replaced by $\mathcal{Z}_{5}$ symmetry (giving an all order hierarchy. See sect. 2.2). For the latter case in the Yukawa superpotential (28) instead of $\epsilon_{m n}$ tensors some arbitrary couplings will appear. It is important that also within this scenario additional vector like states can be decoupled at scale $v_{0}$ and that at low energies we remain with the chiral content of MSSM. Therefore, we can conclude that within an orbifold $S U(6)$ GUT, augmented with additional symmetries, the fermion sector can be naturally constructed.

\section{Gauge coupling unification}

The unification of the three gauge couplings in this scenario occurs near the scale $M_{G} \sim$ $10^{16} \mathrm{GeV}$. The first step of GUT symmetry breaking occurs at the compactification scale $\mu_{0}=1 / R \lesssim M_{G}$. Then below the scale $v_{0}$ we have $G_{321}$ gauge symmetry with MSSM content. Since at the scale $v_{0}$ some vector-like states decouple [see (29), (30)], between $v_{0}$ and $M_{G}$ the b-factors of zero modes will be

$$
\left(b_{3}, b_{3 L}, b_{U(1)}\right)^{v_{0}}=(0,2,10)
$$

Above the $\mu_{0}$ scale, the KK states will contribute into the running and the corresponding factors are

$$
\begin{gathered}
\left(\gamma_{3}, \gamma_{3 L}, \gamma_{U(1)}\right)=(-6,-2,2)+6 \eta \cdot(1,1,1) \\
\left(\delta_{3}, \delta_{3 L}, \delta_{U(1)}\right)=(-2,-6,-10)+6 \eta \cdot(1,1,1)
\end{gathered}
$$

where $\eta$ is number of generations living in the bulk, $\gamma$ and $\delta$ correspond to KK states with masses $(2 n+2) \mu_{0}$ and $(2 n+1) \mu_{0}$, resp. This kind of mass spectrum is due to the $Z_{2} \times Z_{2}^{\prime}$

\footnotetext{
${ }^{6}$ The operator in (31) also can be obtained by exchange of appropriate vector-like states with masses $\sim M$.
} 
orbifold parity prescriptions of (3), (6), (26). Note that above the compactification scale $\mu_{0}$ we consider the effective four dimensional gauge theory with a limited tower of states [16]. Taking these into account, we obtain for the solution of the 1-loop RGE $\mathrm{d}$

$$
\begin{gathered}
\alpha_{3}^{-1}=\frac{12}{7} \alpha_{2}^{-1}-\frac{5}{7} \alpha_{1}^{-1}+\frac{9}{7 \pi} \ln \frac{M_{G}}{v_{0}}+\frac{6}{7 \pi}\left(S_{2}-S_{1}\right), \\
\ln \frac{v_{0}}{M_{Z}}=\frac{5 \pi}{14}\left(\alpha_{1}^{-1}-\alpha_{2}^{-1}\right)-\frac{8}{7} \ln \frac{M_{G}}{v_{0}}+\frac{4}{7}\left(S_{2}-S_{1}\right), \\
\alpha_{G}^{-1}=\alpha_{2}^{-1}-\frac{1}{2 \pi} \ln \frac{v_{0}}{M_{Z}}-\frac{1}{\pi} \ln \frac{M_{G}}{v_{0}}+\frac{1-3 \eta}{\pi} S_{1}+\frac{3-3 \eta}{\pi} S_{2},
\end{gathered}
$$

where

$$
S_{1}=\sum_{n=0}^{N} \ln \frac{M_{G}}{(2 n+2) \mu_{0}}, \quad S_{2}=\sum_{n=0}^{N^{\prime}} \ln \frac{M_{G}}{(2 n+1) \mu_{0}} .
$$

In (37), $N$ and $N^{\prime}$ are the maximal numbers of appropriate KK states which lie below $M_{G}$, i.e. $(2 N+2) \mu_{0} \lesssim M_{G},\left(2 N^{\prime}+1\right) \mu_{0} \lesssim M_{G}$. States which have masses above the $M_{G}$ are not relevant for unification and therefore we truncate the sums in (37) at certain $N$, $N^{\prime}$ numbers of states.

From (34) we see that in order to have a reasonable value of $\alpha_{3}$ we need $v_{0} \simeq M_{G}$ and therefore $\mu_{0} \sim M_{G}$. As one can easily see from (35), the unification scale is close to $10^{16} \mathrm{GeV}$.

\section{Conclusions}

We have presented a 5D SUSY $S U(6)$ GUT construction on an $S^{(1)} / Z_{2} \times Z_{2}^{\prime}$ orbifold. Such a construction together with an additional symmetry suggests a very economical way for understanding the right size of $\mu$ and $B \mu$ terms. With a $S U(2)_{\text {cus }}$ symmetry the Higgs doublets are pseudo-Goldstone Bosons emerging as a consequence of the spontaneous breakdown of a global pseudo-symmetry. We have also shown that alternatively the extension with a $Z_{5}$ discrete symmetry leads to a natural all order hierarchy and to the understanding of the origin of the GUT scale, which is an interplay of $M_{P l}$ and the SUSY scale $m \sim 1 \mathrm{TeV}$. In both types of extensions $\mu$ and $B \mu$ emerge dynamically after SUSY breaking.

\section{Acknowledgement}

F.P.C. is supported by Fundação de Ciência e Tecnologia (grant SFRH/BD/4973/2001).

\footnotetext{
${ }^{7}$ For RGEs with presence of limited KK states see [16], while detailed derivation of RGEs with intermediate scale and KK states can be found in [7].
} 


\section{References}

[1] Y. Kawamura, Prog. Theor. Phys. 105 (2001) 999; ibid. 105 (2001) 691.

[2] G. Altarelli, F. Feruglio, hep-ph/0102301; A. Kobakhidze, hep-ph/0102323.

[3] L. Hall, Y. Nomura, Phys. Rev. D 64 (2001) 055003; M. Kakizaki, M. Yamaguchi, hep-ph/0104103; A. Hebecker, J. March-Russel, Nucl. Phys. B 613 (2001) 3; R. Barbieri, L. Hall, Y. Nomura, hep-th/0107004; C. Csaki, G. Kribs, J. Terning, hepph/0107266; N. Maru, hep-ph/0108002; Q. Shafi, Z. Tavartkiladze, hep-ph/0108247; T. Li, hep-ph/0108120; hep-th/0110065.

[4] T. Asaka, W. Buchmüller, L. Covi, hep-ph/0108021; L. Hall, Y. Nomura, T. Okui, D. Smith, hep-ph/0108071; R. Dermisek, A. Mafi, hep-ph/0108139; N. Haba, T. Kondo, Y. Shimizu, hep-ph/0112132, hep-ph/0202191.

[5] G. Giudice, A. Masiero, Phys. Lett. B 206 (1988) 480.

[6] S. Dimopoulos, G. Dvali, R. Rattazzi, Phys. Lett. B 413 (1997) 336; E.J. Chun, Phys. Rev. D 59 (1999) 015011; P. Langacker, N. Polonsky, J. Wang, Phys. Rev. D 60 (1999) 115005; T. Han, D. Marfatia, R.J. Zhang, Phys. Rev. D 61 (2000) 013007; K. Choi, H.D. Kim, Phys. Rev. D 61 (2000) 015010; Q. Shafi, Z. Tavartkiladze, Nucl. Phys. B 580 (2000) 83.

[7] F. Paccetti Correia, M.G. Schmidt, Z. Tavartkiladze, hep-ph/0204080.

[8] K. Inoue, A. Kakuto, T. Takano, Progr. Theor. Phys. 75 (1986) 664; A. Anselm, A. Johansen, Phys. Lett. B200 (1988) 331; Z. Berezhiani, G. Dvali, Sov. Lebedev Institute Reports 5 (1989) 55.

[9] R. Barbieri, G. Dvali, A. Strumia, Nucl. Phys. B391 (1993) 487; R. Barbieri, G. Dvali, M. Moretti, Phys. Lett. B312 (1993) 137; R. Barbieri et al., Nucl.Phys. B432 (1994) 49; Z. Berezhiani, Phys. Lett. B355 (1995) 481; Z. Berezhiani, C. Csaki, L. Randall, Nucl. Phys. B444 (1995) 61; G. Dvali, S. Pokorski, Phys. Rev. Lett. 78 (1997) 807; Q. Shafi, Z. Tavartkiladze, Nucl. Phys. B 552 (1999) 67; Nucl. Phys. B 573 (2000) 40; B. Bajc, I. Gogoladze, R. Guevara, G. Senjanovic, Phys. Lett. B 525 (2002) 189.

[10] G. Dvali, Q. Shafi, Phys. Lett. B 326 (1994) 258; B 339 (1994) 241; G. Lazarides, C. Panagiotakopoulos, Q. Shafi, Phys. Lett. B 315 (1993) 325.

[11] $2^{\text {nd }}$ ref. in [9]; C. Csaki, L. Randall, Nucl. Phys. B 466 (1996) 41; B.Ananthanarayan, Q. Shafi, Phys. Rev. D 54 (1996) 3488. 
[12] G. Dvali, Phys. Lett. B 324 (1994) 59.

[13] I. Gogoladze, A. Kobakhidze, Z. Tavartkiladze, Phys. Lett. B 372 (1996) 246; A. Kobakhidze, Phys. Lett. B 391 (1997) 335; Z. Tavartkiladze, Phys. Lett. B 392 (1997) 360; I. Gogoladze, hep-ph/9612365.

[14] R. Barbieri, S. Ferrara, C. Savoy, Phys. Lett. B 119 (1983) 123; H.P. Nilles, M. Srednicki, D. Wyler, Phys. Lett. B 120 (1983) 346; L.J. Hall, J. Lykken, S. Weinberg, Phys. Rev. D 27 (1983) 2359.

[15] L. Ibanez, G. Ross, Phys. Lett. B 110 (1982) 215; K. Inoue et al., Prog. Theor. Phys. 68 (1982) 927; Y. Okada, M. Yamaguchi, T. Yanagida, Phys. Lett. B 62 (1991) 54; H. Haber, R. Hempfing, Phys. Rev. Lett. 66 (1991) 1815; J. Ellis, G. Ridolfi, F. Zwirner, Phys. Lett. B 257 (1991) 83; R. Barbieri, M. Frigeni, F. Caravaglios, Phys. Lett. B 258 (1991) 167.

[16] K. Dines, E. Dudas, T. Gherghetta, Nucl. Phys. B 537 (1999) 47. 